\title{
Multi-Agent Information Fusion System to manage data from a WSN in a residential home
}

\author{
Sara Rodríguez ${ }^{\mathrm{a}, *}$, Juan F. De Paz ${ }^{\mathrm{a}}$, Gabriel Villarrubia ${ }^{\mathrm{a}}$, Carolina Zato ${ }^{\mathrm{a}}$, Javier Bajo ${ }^{\mathrm{b}}$, Juan M. Corchado ${ }^{\mathrm{a}}$ \\ a Departamento Informática y Automática, Universidad de Salamanca, Plaza de la Merced s/n, 37008 Salamanca, Spain \\ ${ }^{\mathrm{b}}$ Departamento de Inteligencia Artificial, Facultad de Informática, Universidad Politécnica de Madrid, Campus Montegancedo, Boadilla del Monte, Madrid 28660, Spain
}

\section{A R T I C L E I N F O}

Article history:

Available online 26 March 2014

\section{Keywords:}

Multi-Agent Systems

Wireless Sensor Networks

Information fusion

Ambient Intelligence

\begin{abstract}
A B S T R A C T
With the increase of intelligent systems based on Multi-Agent Systems (MAS) and the use of Wireless Sensor Networks (WSN) in context-aware scenarios, information fusion has become an essential part of this kind of systems where the information is distributed among nodes or agents. This paper presents a new MAS specially designed to manage data from WSNs, which was tested in a residential home for the elderly. The proposed MAS architecture is based on virtual organizations, and incorporates social behaviors to improve the information fusion processes. The data that the system manages and analyzes correspond to the actual data of the activities of a resident. Data is collected as the information event counts detected by the sensors in a specific time interval, typically one day. We have designed a system that improves the quality of life of dependant people, especially elderly, by fusioning data obtained by multiple sensors and information of their daily activities. The high development of systems that extract and store information make essential to improve the mechanisms to deal with the avalanche of context data. In our case, the MAS approach results appropriated because each agent can represent an autonomous entity with different capabilities and offering different services but collaborating among them. Several tests have been performed to evaluate this platform and preliminary results and the conclusions are presented in this paper.
\end{abstract}

(c) 2014 Elsevier B.V. All rights reserved.

\section{Introduction}

Currently, there is a growing need to find more effective ways to provide social services and medical care to the growing number of elderly people with some kind of dependency [1]. This problem has become one of the great challenges for Europe and its scientific community. Some developments such as the AAL (Ambient Assisted Living), sponsored by the IST (Information Society Technologies) under the Seventh Framework Programme of the European Union (European 7th Framework Programme), have focused on finding new ways to address this problem from a technological point of view. As mentioned in [2], Ambient Intelligence is considered "a new way to interact between people and technology, where the latter adapts to individuals and their context and contains a range of interactive devices capable of meeting the demands and requirements of the users". Moreover, it is important to remark that the mechanisms and technologies that form an AmI

\footnotetext{
* Corresponding author.

E-mail addresses: srg@usal.es (S. Rodríguez), fcofds@usal.es (J.F. De Paz), gvg@usal.es (G. Villarrubia), carol_zato@usal.es (C. Zato), jbajo@fi.upm.es (J. Bajo), corchado@usal.es (J.M. Corchado).
}

system must allow their autonomous functioning without disturbing the people's environment but making easy their daily activities.

Information fusion is understood as a process that gathers assessments from the environment. It is based on goals and combines information at a low and high level. As a result, advantages of intelligent approach as Multi-Agent Systems (MAS) within the information fusion process have been recently emerging [3-5]. Agent Technology [6] is gaining progressively more importance in the field of distributed and dynamic intelligent environments; its participation in this process fulfils the requirements and goals of the systems developed under the framework of the Ambient Intelligence and Ambient Assisted Living. These intelligent systems provide a powerful high-level tool and aim to support people in several aspects of their daily life, this support includes the prediction of different dangerous situations, detecting physical problems in people or buildings and also, provide a cognitive support. By integrating intelligent and dynamic mechanisms to learn from past experiences, the proposed architecture is able to provide users with better tools for supplying healthcare.

Wireless Sensor Networks (WSNs) are mainly used to extract information about the environment and behave consequently with an interaction on it, extending users' capabilities and automating 
daily actions [7]. One of the most important applications for WSNs is Real-Time Locating Systems (RTLS). As we previously studied in [8], although outdoor locating is well covered by systems such as the current GPS (Global Positioning System), indoor locating needs still more development, especially with respect to accuracy and low-cost and efficient infrastructures. The use of optimized locating techniques allows obtaining more accurate locations using even fewer sensors and with fewer computational requirements [9].

As presented in [10] the innovative smaller, portable and non-intrusive devices [11] are progressively more efficient when gathering context-information [12]. Thus, the new Ambient Intelligence platforms should encourage the integration of such devices in order to create open, flexible and adaptable systems. This reason lead us to said that virtual organizations of agents are an ideal option to create and develop the open and heterogeneous systems such as those normally found in the information fusion process.

The system represented in this article arises from an increasing need to research new solutions to meet the special needs of the elderly, which will result in one of the most benefit segment of population. The costs associated with these actions are no longer bearable in terms of the investments in infrastructure and human resources required for the traditional model (its survival is not guaranteed). Innovative solutions are therefore needed in order to better address the problem of aging.

More specifically, this paper presents an intelligent multi-agent system aimed at improving healthcare and assistance to elderly and dependent people in geriatric residences and at their homes. The system is based on the PANGEA multi-agent architecture (Platform for Automatic coNstruction of orGanizations of intElligents Agents) [13], which provides a high-level framework for intelligent information fusion and management. The system makes use of wireless sensor networks and a Real-Time Locating System to obtain heterogeneous data, and provide autonomous responses according to the environment status. The proposed system integrates a set of autonomous reactive and deliberative agents designed to support the carergivers' activities and to ensure that the patients are given the proper care in both the residence and their homes.

In summary, our work consists on developing a good tested system for information fusion technologies in an important real-world application scenario, covering the following issues:

- The development of a new Ambient Intelligence based multiagent system aimed at improving the healthcare of dependent people in geriatric residences and in their own homes, focusing on information fusion techniques and extending the system proposed in [1].

- The development under the PANGEA multi-agent architecture, which provides a high-level framework for intelligent information fusion and management.

- The appropriated use of virtual organizations of agents for the overall management; and control systems for high-level sensor data management.

- To include a rule-based reasoning system to improve the accuracy of the results.

The use of virtual organizations of agents facilitate the incorporation of new information fusion techniques to the platform. Selfadaptive virtual organizations allow the dynamic incorporation of specialized agents, which provides a framework for the incorporation of new information fusion techniques. The remainder of the article is structured as follows: sections two and three review the state of the art of related projects, both national and international, as well as the various types of technology used in the study (information fusion, agents, rule-based reasoning systems, case based reasoning), in an effort to identify current deficiencies. Section four presents the proposed multiagent system, providing a general description, its infrastructure and integration within sensor networks. This section specifically describes the basic structure of the developed system, which is composed of three distinct parts: real time identification and localization service [1]; telemonitoring services; and an interface service for personnel. This article focuses on telemonitoring services that permit carrying out a study, and the observation and analysis of the users, which in this case are the elderly living either in a care home or in their own home. Finally, section five presents the results and conclusions obtained from the study.

\section{State of the art}

The emergence of new technologies had resulted in a number of projects that aim to improve interaction with an environment. One specific application involves improving quality of life and care of the elderly, as noted in the following specific projects:

- The CommonWell European program [14] proposes an architecture to support citizens with limited mobility or an audio or visual impairment. It focuses on the elderly but does not incorporate as many advanced adaptable or identification interfaces or location.

- The European DTV4A project proposes the use of digital television to integrate persons with limited abilities; however, the television is the only mechanism to provide the services and obtain information.

- The European Monami project [15] proposes a global framework to offer services to the elderly and disabled. It focuses on providing these services to individuals with the aim of enabling a more independent lifestyle. It does not, however, provide information for medical personnel or an alarm system.

At the national level we find the following:

- The DISCATEL project [16] aims to facilitate contact with disabled people. It offers a monitoring system especially for people with disabilities.

- The INREDIS (Interfaces for Relations between the Environment and people with disabilities) Project [17] is a CENIT project led by Technosite, which combines the concept of using personal devices with ubiquitous interoperability and characteristics to strengthen accessibility for people with special needs.

- The INCLUTEC group [18] is developing the eVia platform, which is oriented toward the analysis and development of new mechanisms to facilitate mobility such as advanced wheelchairs and specialized assistance vehicles, alternative and improved communication, manipulation and cognition. Although it can be applied to the elderly sector as a whole, this project also focuses on persons with disabilities.

Apart from the projects in development, there are a high number of projects involving wearable health devices [19-21] integrated with sensors providing continuous monitoring of person's health related issues and daily activities. There are also many systems developed to monitoring and with the recovered information, create alarms and facilitate the clinical decisions, i.e. Arezzo, DeGeL, GLARE, GLEE, HeCaSe2 compared in [22]. SHAPHIRE [23] is a system developed to provide clinical decision support for remote monitoring of patients at their homes and at the hospital to reduce the load of medical practitioners and healthcare costs. The system CAMPH, a context-aware middleware for pervasive homecare, is presented in [24]. The middleware offers several 
key-enabling system services that consist of P2P-based context query processing, context reasoning for activity recognition and context-aware service management. However, camera based sensors for surveillance and security in which the images of the person are taken require acceptability of the elderly which may not be possible. BehaviorScope [25] processes streams of timestamped sensor data along with prior context information to infer activities and generate appropriate notifications. In this case, the activities of interest are pre-programmed into a specification that is used by the system to interpret the incoming sensor data stream. The system interprets the activities to generate summaries and other triggered notifications that are propagated to the users. However, these projects are for very specific purposes.

There are also works centered on improving the activity patters of the elderly, looking for a better learning of the software. This is the case of the framework called DTFRA (Discovering of Temporal Features and Relation of Activities), which focus on discovering and representing the temporal features of activity patterns from sensor data. The proposed algorithm is able to discover features and relations, such as the order of the activities, their usual start times and durations by using rule mining and clustering techniques. Other related works focus on this specific activity are [26-28]. In some cases, these systems are extended with monitoring and modeling the activities of daily living $[29,30]$.

The most complete system found in our previous research was JTH [31]. It is a prototype of wireless sensor networks, an interconnection platform and a service management platform to support large scale data interconnections and real-time activity and health state reports to related persons (e.g. doctors or nurses, elder-self, relatives) via all popular communication approaches, such as automatic voice telephone call, SMS or Email etc. This system provides 4 main functionalities, including indoor monitoring, outdoor monitoring, activity and health state decision, emergency decision and alarm.

The survey [32] about wireless sensor networks for healthcare presents some interesting systems related to the field. Nevertheless, our system is technologically far away from those taken into account. This system is more than a decision support system since it integrates other capacities such as location, monitoring and prediction of dangerous situations. Moreover, it is based on the multiagent technology, which is highly appropriate to this end due to the need of fusioning information from heterogeneous distributed resources and autonomous entities. In this sense, the main contribution of this work is a system easily extensible that conforms a complete tool for health-care services based on WSN sensors networks. The skeleton of the system is the PANGEA platform, which provides the basic characteristics for the perfect functioning of the agents.

What we are presenting is a system that aims to improve the action of assistance in a senior home care facility. The system as a whole offers real time identification and location services [1], telemonitoring, and interface services for personnel. To this end, techniques such as information fusion, rule-based reasoning systems, WSN and MAS are used. These techniques are presented below.

\subsection{Information fusion and agents}

Most of the times, information fusion is a fundamental part of sensor management. Waltz in [33] define sensor management as "any system which provides automatic control of a suite of sensors or measurement devices and its. The main problem has to do with the ability to observe a dynamic scene with a set of sensors by controlling their configuration, their sequencing or their state changes, as well as the scheduling of the resources". Moreover, information obtained from multiple sensors needs to be fused because no single sensor can get all the information, and the information from different sensors may be uncertain, inaccurate, or even conflicting [34].

In our previous work [35] has been already exposed that there is a considerable variety of sensors that can observe user contexts and behaviors and multi-agent architectures that utilize data merging to improve their output and efficiency. Such is the case with Castanedo et al. [36], Pfeffer et al. [5], Liu et al. [37] or the system called HiLIFE [38].

Multiagent architectures are specially appropriated to implement the new algorithms for information fusion and to manage high level information. This adequacy of agents and multi-agent systems applied to information fusion has been deeper discussed our previous work [39]. Open MAS [40] and virtual organizations of agents [41-44,40,45], as a specialized version of MAS, are used in this paper to allow the inclusion of organizational concepts These concepts includes rules and norms [46], groups or institutions [47] and social structures [48].

From our perspective, there is already an open platform that has been created and allows any type of configuration, adaptation mechanisms, reorganization, search services, etc.

\subsection{Rule-based reasoning systems}

As observed in [49], the most critical step in information fusion is related to the transformation from the observed parameters, which are obtained by multiple sensors, and a decision or inference, which is calculated by fusion estimation and/or inference processes. Finally, it is also important to get an easy understandable interpretation of the observed situations and the relationships between them. In many cases, this interpretation needs not only implicit information but also explicit data that must be extracted via knowledge-based methods such as rule-based reasoning systems.

The goal in building an automated reasoning system is to create a system capable of making decisions based on the information obtained through the heterogeneous sensors.

The developed system (and the agents supporting it) uses very different techniques for base and meta-classifiers training as will be seen in Section 4. They are used in the extraction of data and the association rules from different sources of information. The system applies information fusion algorithms which combine the information gathered from each of the sensors through a casebased reasoning (CBR) mechanism [50]. But, in most cases, CBR has not been used alone, but combined with various artificial intelligence techniques. Support Vector Machine (SVM) [51] has been used with CBR in this study to perform the classification of the data obtained by sensors and automatically create the intern structure of the case base from existing data.

\section{System overview}

In this paper we propose a new Ambient Intelligence based multi-agent system aimed at improving the healthcare of dependent people in geriatric residences and in their own homes, focusing on information fusion techniques and extending the system proposed in [1]. The most important issue in this system is the application of information fusion algorithms [52-54] that manage data from Wireless Sensor Networks (WSN). The data collection is carried out in real-time according to the action that occurs in the environment, due to this, the agents can react accordingly in an automatic and instantaneous way. Thanks to this configuration the system enables the integration of an elevated number of WSNs with the advantage of a greater simplicity due to the reuse of available resources. 
The agents in the system are implemented within the agent platform PANGEA (Platform for Automatic coNstruction of orGanizations of intElligents Agents) [55,13]. PANGEA helps the system to create agents in charge of all the functions of the system, allowing them to organize and communicate more easily and securely, regardless of how they are created, where they are located, what data to collect or what role they play within the system.

The two key concepts that come together in this system are: the use of virtual organizations of agents for the overall management; and control systems for high-level sensor data management. Furthermore, the sensors themselves will have to be managed and analyzed to extract information from them and apply them to the case study in question (healthcare and assistance to elderly and dependent people in geriatric residences).

The context information includes not only information about the environment, the people who lives in such environments is also monitorized. This information includes parameters such as location, temperature of the building, quality of the air, and heart rhythm of the patient.

A node is each element that is included in a sensor network. Each sensor node is usually formed by a microcontroller, a transceiver for radio or cable transmission, and a sensor or actuator mechanism [11]. Some nodes act as routers, allowing them to forward data that must be delivered to other nodes in the network. There are wireless technologies such as Wi-Fi, IEEE 802.15.4/ZigBee and Bluetooth that enable easier deployments than wired sensor networks [7]. At the level sensors, the basis of the WSN infrastructure of the system is made up of several ZigBee nodes. The ZigBee standard features make ZigBee an ideal supporting wireless technology for building indoor Real-Time Locating Systems. The possibility of working with low-power nodes that do not need large computational resources allows designers to reduce hardware costs when implementing the systems. In addition, these kinds of low-power nodes can reach a battery life of several years, with regards to the transmission range (transmitted power), the time resolution and the accuracy of the system. ZigBee-based Real-Time Locating Systems can use different locating techniques in order to estimate the positions of the tags in the environment. In the proposed system, each ZigBee node includes an 8-bit RISC (Atmel ATmega 1281) microcontroller with $8 \mathrm{~KB}$ of RAM, $4 \mathrm{~KB}$ of EEPROM and $128 \mathrm{~KB}$ of Flash memory and an IEEE 802.15.4/ZigBee transceiver (Atmel AT86RF230). These devices, called n-Core Sirius B and Sirius D are shown in Fig. 1 [56].

At higher levels (features and decision), it is possible to detect alterations in the environment and its corresponding response. For example, if a change within a node (a change of light for instance) is detected at the sensor level, the agents at a higher level can decide to send a warning message or perform an action. The actions and reactions are handled by the PANGEA platform since all the agents that formed the organizations are designed with the corresponding services and functions.
This configuration enables the capacity to manage a variety of sensors, other devices of diagnostic and different sources of information (maintenance records, monitoring and observations). The framework provides for information synchronization and highlevel fusion [57].

\subsection{Infrastructure}

The basic infrastructure of the system proposed in this article consists of the following elements:

Wireless sensor infrastructure. This infrastructure is the foundation of the main system services. The infrastructure consists of a set of physical wireless devices on which part of the low level middleware will be executed and through which the rest of the system can access its functionalities (for example, obtaining readings from various sensors or calculating the location of the user).

Wireless devices. They form part of the wireless infrastructure hardware. A set of low consumption and small wireless devices were deployed. Each one shares a common basic architecture composed of a microcontroller, a transceptor and a set of physical interfaces for the exchange of data between the device itself and the sensors and actuators to which the device is connected. According to the application, these devices can be either battery or externally powered.

Low level middleware. A low level middleware is executed on the wireless sensor network. It is composed of different layers that permit the exchange of data between the wireless devices and the other system components. The low level middleware contains a multiagent system $[1,13,58]$ which permits the exchange of information between the firmware included in the wireless devices, the API communication to access the functionalities, and the communication protocol.

API communication. The API (Application Programming Interface) allows the rest of the system to interact with the wireless devices in order to gather the information taken from the sensors they are connected to, and to send and receive data.

Agent-based infrastructure. This infrastructure, together with the wireless sensor infrastructure, is the other pillar of the system. It will be supported by PANGEA and will enable the transfer of tasks that require a high computational cost and that, as a result, are very difficult to implement in machines and devices, often mobiles, used by the system clients. This infrastructure is essentially composed of the agents that provide the system functionality and that can be found in a remote server. The agents execute the tasks (e.g., location). PANGEA also provides the network communication data infrastructure, which makes it possible to connect with client machines and for agents to communicate with the services they offer.

Graphical interfaces. Last, but not least important, the system includes a set of graphical interfaces that can display an

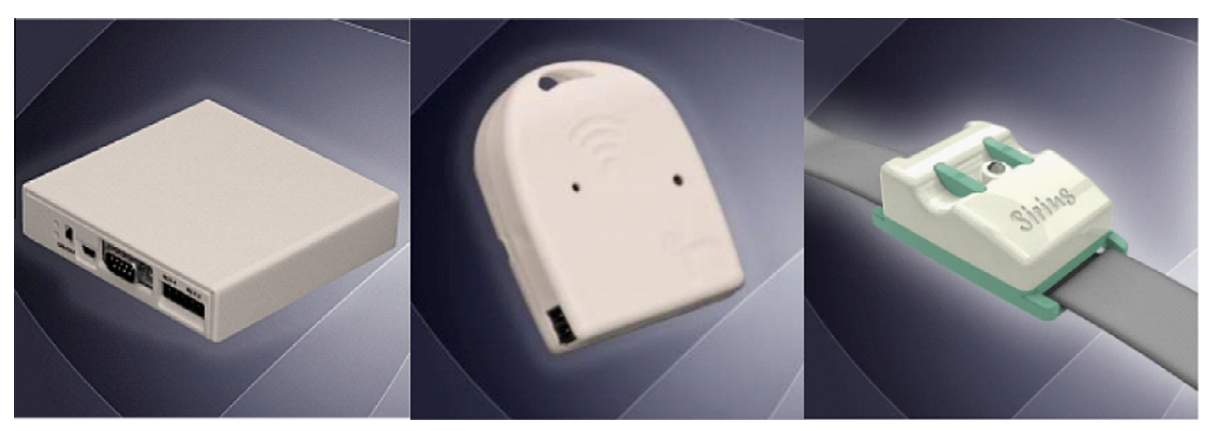

Fig. 1. n-Core Sirius devices. 


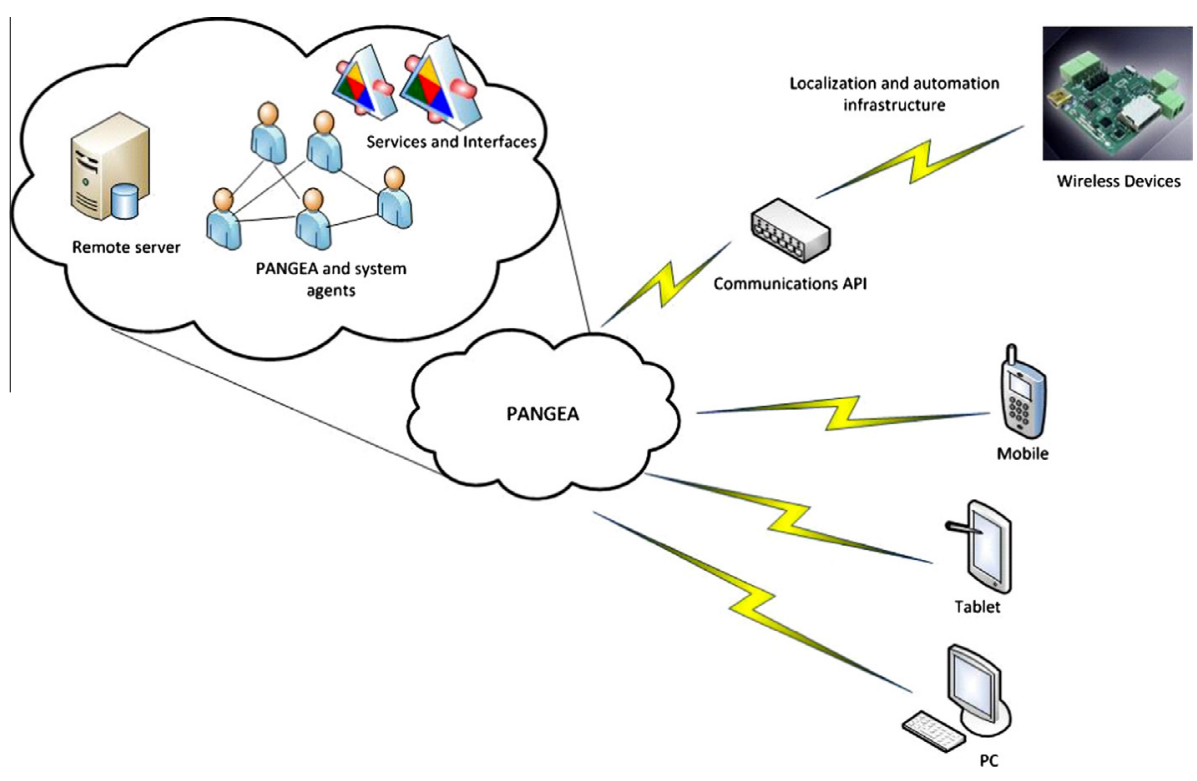

Fig. 2. System architecture.

enriched form of all the information provided by the agents based on the data they have received. This makes it possible to access all the system information and the systems themselves using practically any device that can execute a simple web browser (see Fig. 2).

All the artifacts, including sensors that make up the system have been developed and combined with PANGEA platform to obtain a system specifically oriented to manage information obtained of the environment. For the communication between agents, the IRC (Internet Relay Chat) Protocol is used in PANGEA platform [55] demonstrating its robustness. IRC is a real time internet protocol for simultaneous text messaging or conferencing. IRC provides advantages, such as ease of implementation and reliability, given that it has been widely used in online societies with good functionality. It is used mainly for agent's communication but also allows private messaging for one on one communications, and information transfers. All messages are the following format (see Fig. 3):

The prefix is voluntary in some messages; the command is one of the originals from the IRC standard. For example (see Fig. 4):

All agents that are created in PANGEA extend a common basic structure that supports sending and receiving these messages. This basic agent has implemented basic methods like "initialize" or "execute" which initializes the agent with some basic information, or begin the implementation, respectively. A platform's code generating tool makes it possible to easily create an outline of an agent, with the communication code requiring few lines of code. The following lines of code are an example of this (see Fig. 5).

The architecture was initially designed to be applied within medical care environments, and defined three types of services that can be easily adapted to other types of environment:

- Real Time Identification and Location Service: system for locating elderly or dependant people and staff.

- Telemonitoring service: system with different kind of sensors that fusion the information obtaining knowledgment and alerts.

- Caregiver support system: system that allows the caregivers to set alarms and plan daily tasks and routines to take care of the elderly.

\section{prefix command command-parameters $\backslash r \backslash n$.}

Fig. 3. Message format

PRIVMSG < HomeAutomationorganization:text>

Fig. 4. Example of message

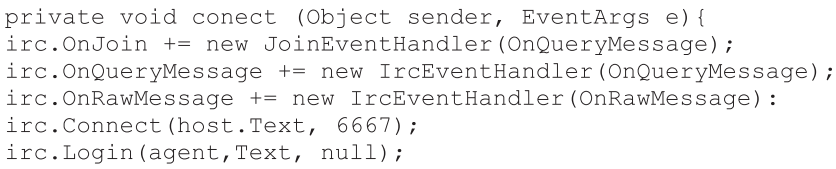

Fig. 5. Agent communication code.

The two first services are explained below in greater detail, explaining how they are offered through the agents in the PANGEA system.

\subsection{Real Time Identification and Location Service}

The Real Time Identification and Locating Service can identify and know the position of the user or a particular object at any given time. As with other services, it can also use algorithms to manage alerts related to the location of the users and objects in relation to the area in which they are found, permission, etc. It does so by employing a set of location algorithms [52-54], which provides greater precision in locating persons than current location systems. These algorithms fuse the information gathered from different sensors: ZigBee, Wi-Fi, accelerometers and compasses.

The configuration used in the system is similar to the configuration of our previous and well tested system presented in [1]. It consists of a ZigBee tag mounted on a bracelet worn on the users' wrist or ankle, several ZigBee readers installed throughout protected zones, and a central workstation where all the information is processed and stored. These readers are installed all over the facilities so that the system can detect when a user is trying to enter a forbidden area according to the user's permissions profile. The ZigBee 
network also allows obtaining information of the environment from different sensors, such as temperature sensors, light sensors, as well as smoke and gas detectors. In addition, different locating techniques can be used as readers and tags carried by patients and medical personnel. These devices are small enough to be carried by a patient, a caregiver or even an object, and have a battery life of up to six months. The location of users is given as coordinated points obtained from the locating techniques provided by a locating engine [59]. All information obtained by means of these technologies is processed by the PANGEA agents. The system allows users to keep track of any tag in the system as well as receive distinct alerts in real-time coming from the system in any Web-based device, such as PC or a smartphone carried by doctors and nurses. Some of the different alerts include panic button alerts (when users press a panic button on their tag or in a fixed device including such a button), forbidden area alerts (when users enter a forbidden area according to their permissions), as well as low-battery alerts (if a tag in the system should be recharged).

The ZigBee infrastructure was deployed in a $600 \mathrm{~m}^{2}$ area within a residence housing dependent people with distinct types of dementias such as Alzheimer's disease. The locating infrastructure is intended to provide the real-time position of people (i.e., patients and medical personnel) and assets (i.e., wheelchairs and lifters) with an average accuracy of $2 \mathrm{~m}$ within the monitored area in hallways and $0.65 \mathrm{~m}$ inside bedrooms, since these are the areas which contain a greater number of sensors. Fig. 6 shows one of the Web-based interfaces of the telemonitoring system in the monitored area.

In order to avoid problems produced by these algorithms, the location system's start-up procedure is modified. Instead of calculating the position of a tag based on the position of the reader, we instead calculate a map of intensities for the environment. We take the tag and calculate the RSSI levels obtained for each reader in the different areas. Using this procedure, for every point $(x, y)$ in a plan, we obtain a set of measurements represented in:

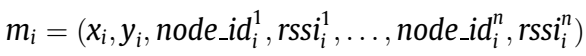

where $m_{i}$ represents the measurement $i, x_{i}, y_{i}$ represent the $x$-coordinates and node_id $j_{i}^{j}$, which is taken from the plans for measurement, $i$ represents the identifier for node $j$ for measurement $i$, and $r s s i_{i}^{j}$ represents the RSSI value of node $\mathrm{j}$ from measurement $i$. Using $m=\bigcup m_{i}$ we can build a classifier based on the data from $m$. The classifier is incorporated into the system's LocationAgent, which is in charge of determining coordinate $x$ according to the values from the RSSI signals obtained from the different readers. The classes are defined according to the different pairs of values $\left(x_{i}, y_{i}\right)$.

We have applied a Bayesian network. There are various Bayesian network search mechanisms, including tabu search [60], conditional independence [61], K2 [60], HillClimber [60], TAN (Tree Argumented Naive Bayes) [62]. We have also used conditional independence, an algorithm based on the calculation of the conditional Independence test for the variables to generate a DAG that can obtain probability estimates. Assuming $r$ pairs of different values for $\left(x_{i}, y_{i}\right)$, the probability of measurement $m$ belonging to class $i$ applying classifier $C$ is defined as follows:

$p_{i}=C\left(m^{\prime}\right)$

where $m$ is the class with $k<r$ if

$p_{k}=\operatorname{Max}\left(p_{i}\right)$ with $i=1 \ldots r$

The algorithm simply places the tag in position $\left(x_{i}, y_{i}\right)$ with greater probability.

\subsection{Telemonitoring service}

The telemonitoring service can gather information from the context and respond by generating alerts and other relevant actions. To do so, it uses the contextual information taken from different sensors and from new knowledge extraction algorithms.

\subsubsection{Rule-based telemonitoring}

The telemonitoring service has the following objectives: (i) monitor through the use of data gathered from sensors for each participant; (ii) use the "Presence of Trace" of each person; (iii) know the different available means of monitoring (devices, professional and support personnel, resources, etc.) and the communication that exists among them, and use the assistance device to suggest certain actions to take; (iv) control and know what is

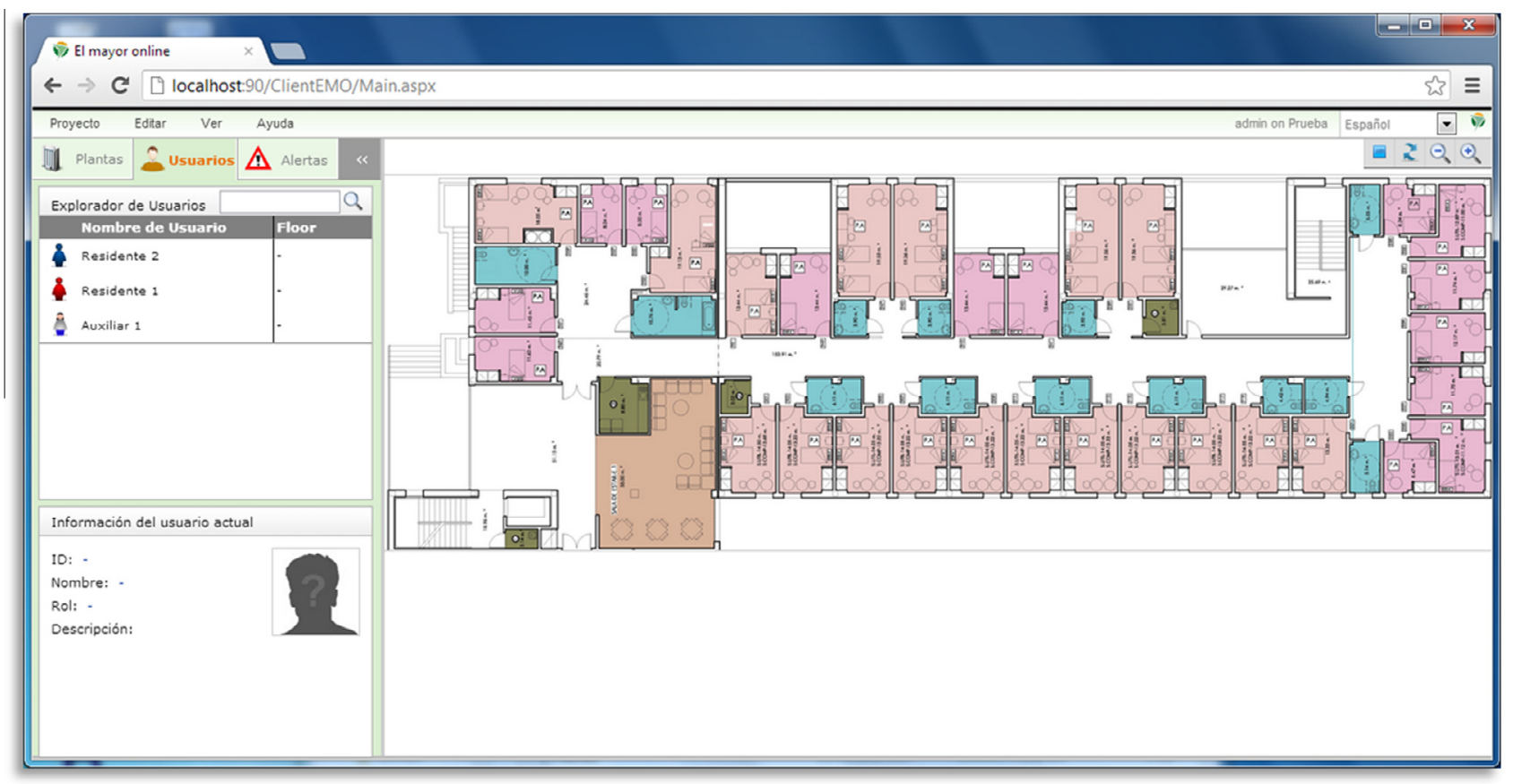

Fig. 6. Software interface of the locating system. 
occurring in the global context of the residence of the person under supervision; and ( $v$ ) add and personalize rules as data are expanded.

The service is offered through a remote monitoring center, a sensor network, wireless actuators deployed within the environment, and a communication network that connects all the system components.

As an example, we have a case study in which medical care is provided in a care facility and private home. This requires an infrastructure of sensors and actuators deployed both in the care facility and the home. The sensors gather information from the context, that is, from both the elderly patient and the environment itself. The information is processed and analyzed by a set of mechanisms that facilitates the decision-making process and optimizes the response of professional and support personnel. Those responsible for assisting can access specific information regarding the elderly patients: physical location in the home, medical history, high risk situations detected, etc. Similarly, after providing assistance, the data gathered from the actions taken are gathered for their subsequent analysis.

The information gathered by the infrastructure deployed in the system is fundamental for the development of a "virtual assistance services" system, which can be personalized for the home of each person. The telemonitoring infrastructure is composed of a set of Wireless ZigBee sensors [63] completely integrated within the environment. The network in the system pilot was deployed in a typical care facility bedroom (simulating the patient's home) as shown in Fig. 7. This space and the full residence were used to carry out the tests.

Fig. 7 shows a map of the deployment of sensors in living quarters. In addition to the ZigBee readers (red squares), and sensors, 4 points (blue circles) were included in the movement pattern node: one associated with the bathroom, one with the bed, one with the dining table, and the other with the front door. The zig bee readers must be located surrounding to the pattern nodes. The presence sensors are located in positions avoiding blind spots. At this point, it is important saying that here an example deployment is shown as each person will have their individualized monitored deployment system and therefore its associated rules. One advantage is that the system can adapt the system rules for each monitored person and the number and type of sensors associated. In the administration tool it is possible to select each of these monitored persons to observe the distribution of deployed sensors. And the rule system is customized through a case-based reasoning as it is explained below.

The alerts generated by the sensors are managed through a multiagent architecture that applies algorithms to manage the information and make decisions. The alarm management system is based on a behavioral module which applies the Drool production rule system, allowing the information to be processed quickly and decidedly. The architecture applies information fusion algorithms [1] which combine the information gathered from each of the sensors installed in the environment and the tracking information for each user to generate intelligent alerts through a casebased reasoning (CBR) mechanism [50]. The system includes a "daily activity planner" and a library of "rules" that generates alerts when something occurs contrary to the typical daily plan. For example: "If the subject remains in bed longer than eight hours, an alert is generated".

The detection of anomalous behaviors is done through the detection of strange movement patterns of the users. In order to detect these patterns, a series of relevance variables are established to measure a series of variables in each of the nodes in the graph that represent possible movement patterns. The values indicated in Table 1 are used for each node representing a movement pattern. The nodes for each movement pattern do not include the sample points in the map of intensities to reduce the number of values in Table 1.

The agent that detects anomalous behaviors is based on the use of predefined rules that are executed over the data from the previous table. An example of the rules can be seen in Table 1 . The rules are written in a text file and they can be modified without building the source code. The Drools production rule system [64] was used to configure the rules engine. Drools is a business rule management system (known as BRMS, Business Rule Management System) with a forward chaining inference based rules engine, using an implementation oriented toward Rete algorithm objects [65]. In other words, it is a rules engine based on Java, responsible for

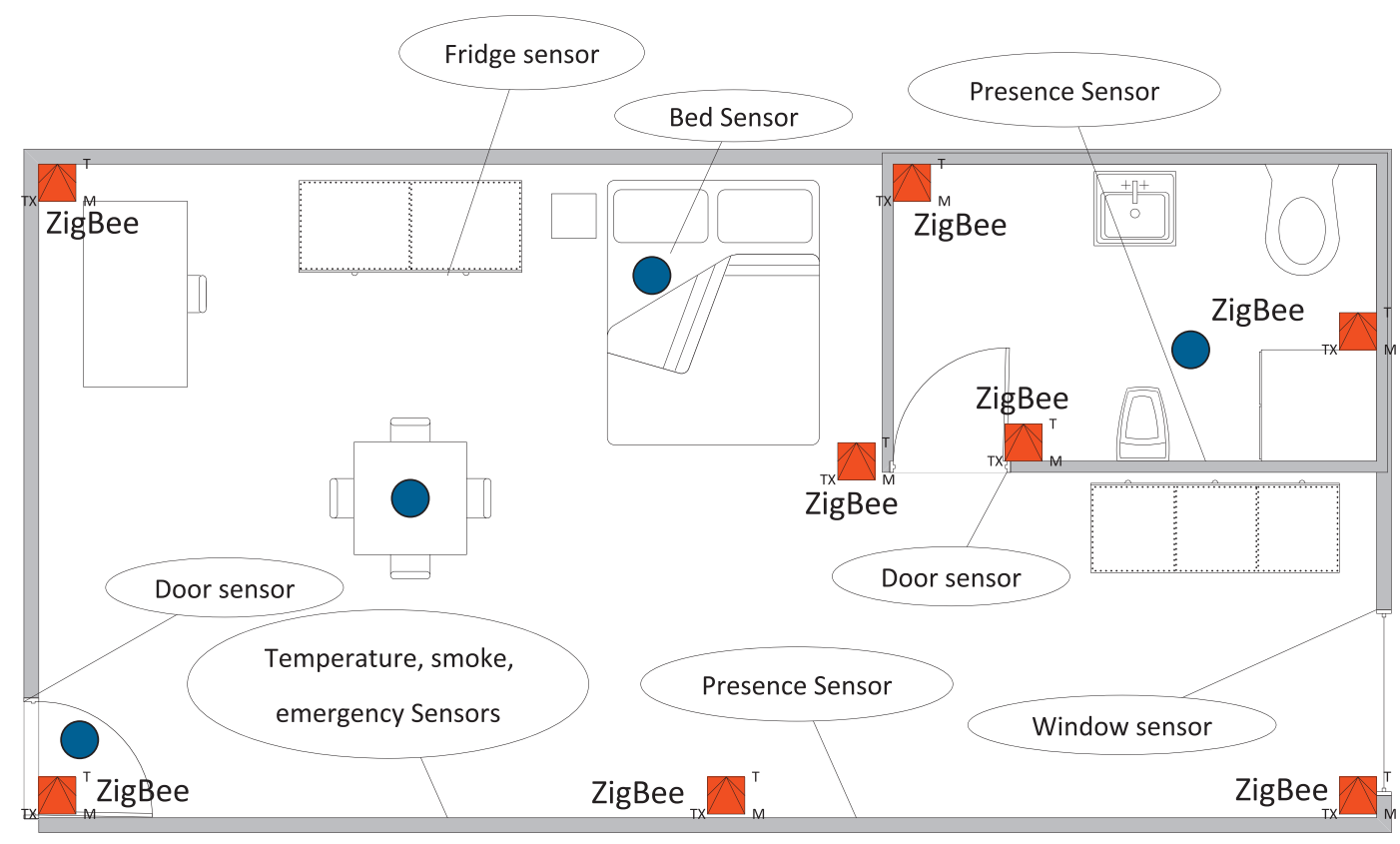

Fig. 7. Map of the deployment of sensors in the living quarters. 
Table 1

Tracking data used. The variables used in Eq. (4) are represented in brackets.

\begin{tabular}{ll} 
Day of week $(\mathrm{w})$ & $1 \ldots 7$ \\
Hour $(\mathrm{h})$ & Seconds starting at 0:00 \\
Node $(\mathrm{n})$ & Current node \\
User $(\mathrm{u})$ & User id \\
Seconds $(\mathrm{s})$ & Seconds from the previous node \\
Previous node $(\mathrm{p})$ & ID of previous node \\
Previous time $(\mathrm{l})$ & Seconds since the node was last accessed \\
Number of times $(\mathrm{t})$ & Number of times user has been in node since 0:00 \\
\hline
\end{tabular}

applying business rules in our applications. This makes it possible to abstract the code and process the objects and a relatively high level with a language that is more simple and closer to natural language, separating rules from actions (see Fig. 8).

The Drool production rule system [64] is also used to manage the information provided by the sensors. The information gathered from the sensors makes it possible to know the state of the environment as well as the time instance in which the change was made and the number of measurements taken in that particular state, as shown in Table 2.

In addition to the previous rules, case based reasoning is used to generate additional rules based on J48 to detect new cases identified as anomalous. If any of the procedures detects anomalous behavior, an alarm will sound, unless a Drool rule to cancel an alarm that has been activated through J48 is detected. The ability to automatically cancel rules is to avoid launching a false positive and allow the automatic cancellation of an alarm considered to be incorrect.

The first step in defining the case-based reasoning is to establish the information according to Tables 1 and 2, as shown in (4). The variables are described in Tables 1 and 2 . The case base is divided into similar groups in order to improve the prediction capability of the system.

$$
\begin{gathered}
c_{i}=\left(w_{i}, h_{i}, n_{i}, u_{i}, s_{i}, p_{i}, l_{i}, t_{i}, D^{i}\right) \\
D^{i}=\bigcup_{j} d_{j}^{i} \\
d_{j}^{i}=\left(i d_{j}, d t_{j}, d s_{j}, d m_{j}, d a_{j}\right)
\end{gathered}
$$

Once the concept of case has been defined, the CBR cycle is defined for each of the following steps: retrieve, reuse, revise and retain. As new cases are received in the system, they are introduced into the reasoning cycle. A prediction is made regarding the need to generate an alarm. The complete process is described in Fig. 9.

The retrieve phase is initiated upon receipt of a new case $c_{n+1}$. When the new case arrives, it is associated or classified with one of the groups $g_{k}$ into which the memory is divided. Once the group
Table 2

Sensor information. The variables used in Eq. (4) are represented in brackets.

\begin{tabular}{ll}
\hline Sensor (id) & Id sensor \\
Time $(\mathrm{dt})$ & Date of last change \\
State $(\mathrm{ds})$ & State of the sensor \\
Measurements $(\mathrm{dm})$ & Number of measurements taken in the state \\
Alarm $(\mathrm{da})$ & Alarm activated \\
\hline
\end{tabular}

is determined, the J48 classifier associated with the set is recovered or created, if it did not previously exist. In order to perform the classification, the SVM [51] is applied, as part of the process indicated in Fig. 10a.

During the revise phase, the classifier retrieved in the previous phase is applied to the new case, and a prediction is obtained.

During the retain phase Fig. 10b it is determined whether the prediction made in the revise phase was satisfactory; if it was not, the prediction is updated. During the revise phase the expert confirms the prediction and during the retain phase the prediction is stored according to a results obtained. That is, the system is capable to detect new cases identified as anomalous, is capable to automatically cancel rules is to avoid launching a false positive of an alarm. In this sense, if in the revise phase the expert confirms that the alarm was a false positive is stored. If the prediction is incorrect, the new case is introduced into the system and the process of clustering and creating a classifier is carried out for each of the clusters created. The EM method is used to create the clusters since it facilitates the creation of groups without requiring the number of clusters to be previously indicated. Additionally, it works with nominal data, a process indicated. Finally, the SVM is constructed according to the new groups and the system constructs the decision tree J48 for each group.

\subsection{Integration within the multiagent system}

PANGEA (Platform for Automatic coNstruction of orGanizations of intElligents Agents) [13] is an agent platform to develop open multi-agent systems that can manage roles, norms, organizations and suborganizations, and facilitate the inclusion of organizational aspects. The PANGEA platform has been adapted to be applied in context-aware environments, with special attention placed on obtaining new algorithms and modules for the fusion of contextual information. This is a new perspective where social aspects are taken into account to model and manage intelligent environments. The basic agent types needed to the adequate functioning of the platform and the main characteristics of it are defined in [55].

Four specialized suborganizations have been created in the platform, all of which are managed by an OrganizationalAgent; this helps the OrganizationManager to control the correct functioning.

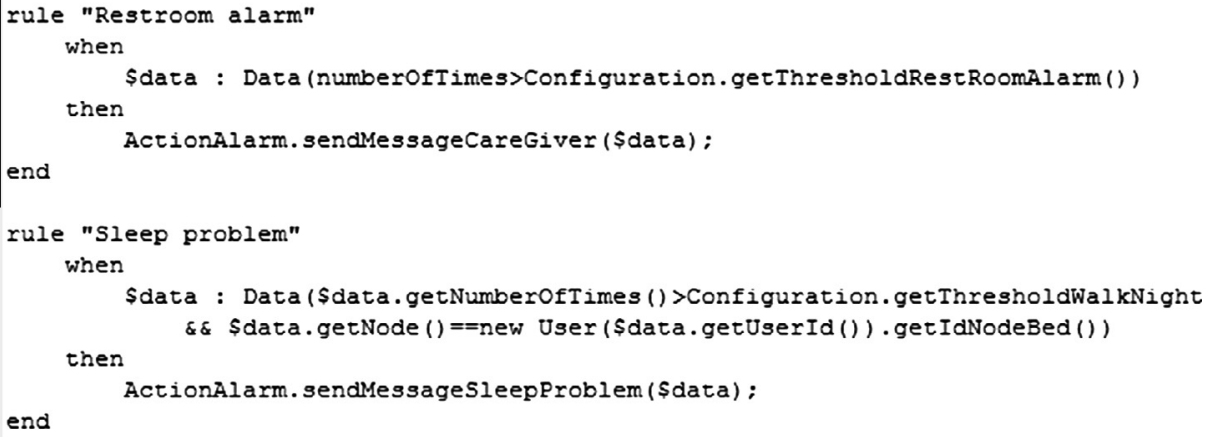

Fig. 8. Example of rules. 


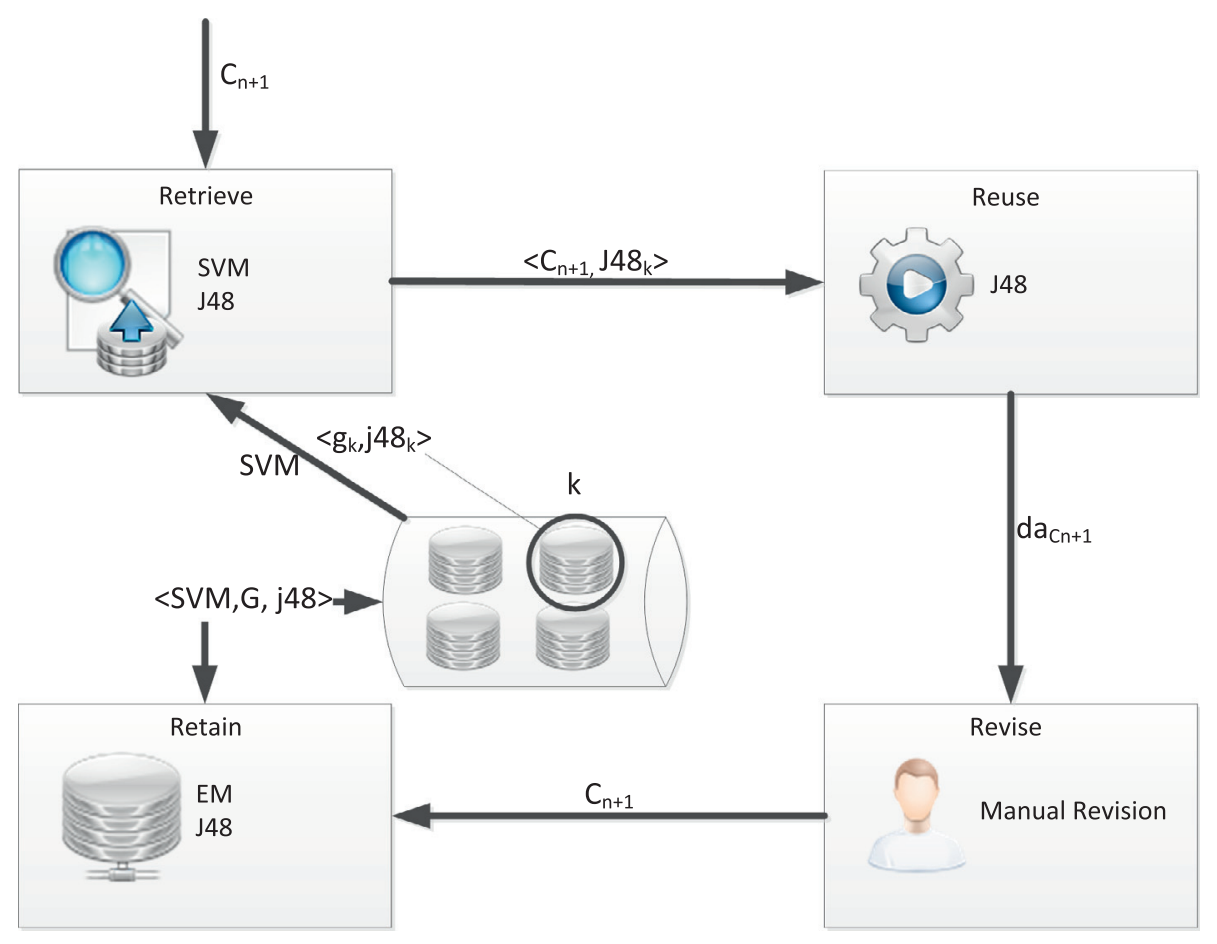

Fig. 9. CBR reasoning cycle.
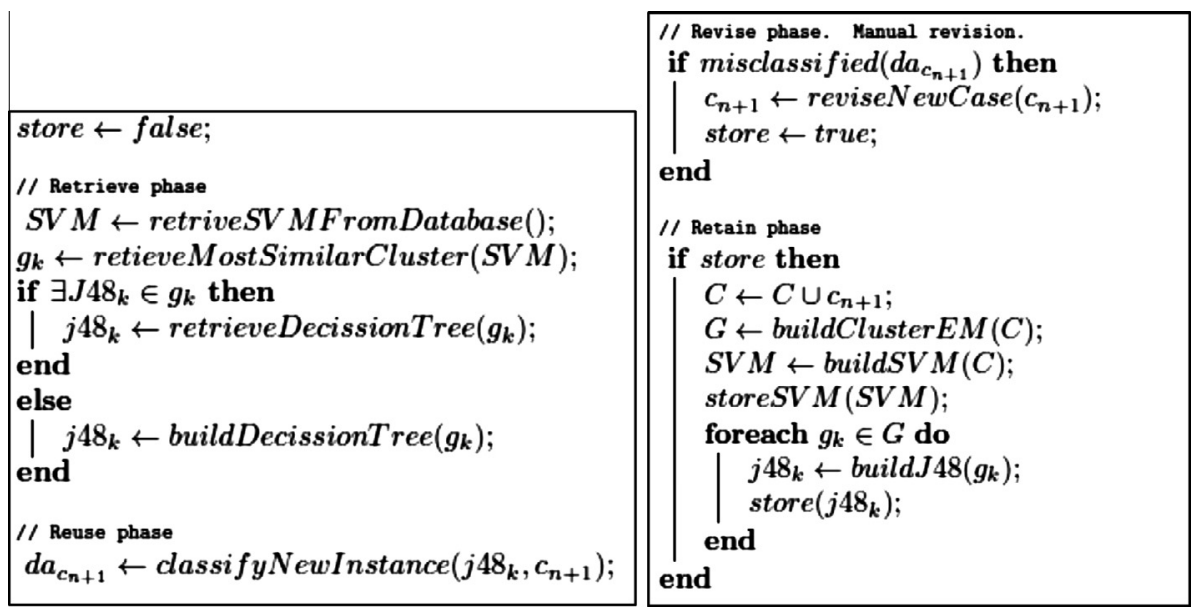

Fig. 10. (a) Algorithm retrieve and reuse phase, (b) revise and retain phase.

Each suborganization is specialized according to the tasks carried out. The four suborganizations, which can be seen in Fig 5 are:

- InformationProviderOrganization: enables interaction with the user. As three different interfaces have been developed, there is an agent for each one of them.

- HomeAutomationOrganization: composed of all the agents that control the different sensors of the spaces (presence, smoke, temperature, etc.). All the information collected by them is sent to the ZigbeeSupervisorAgent. After a previous evaluation, this agent communicates the information to the InformationAgent. The InformationAgent is the interface with the database.

- LocatingOrganization: composed of all the agents that control the Zigbee sensors and tags, which allows locating the people (caregivers or patients). All the information collected by these agents is sent to the ZigbeeSupervisorAgent. This information is used by the MonitorAgent and the LocatingAgent if the user wants to track a person.
- CaregiverOrganization: each caregiver (doctor or nurse) is represented by an agent that collects information related to their respective actions. Each action has a representative collection of information that allows for knowledge extraction. This information is key for the proper operation of RulesAgent As it can, for example, create the routine for each patient. When the information provided by the caregivers and sensors falls outside the patient's routine or indicates a dangerous situation, the AlarmAgent extends the alarm throughout the system. The MonitorAgent can receive the alarm because of its communication with the SnifferAgent and shows it on the screen (see Fig. 11).

\section{Results and conclusions}

As indicated in the previous section, a case study was prepared in a medical care environment for the elderly. The proposed system was implemented in the "El Residencial La Vega" care facility in the city of Salamanca and was tested over a period of 8 months. The El 


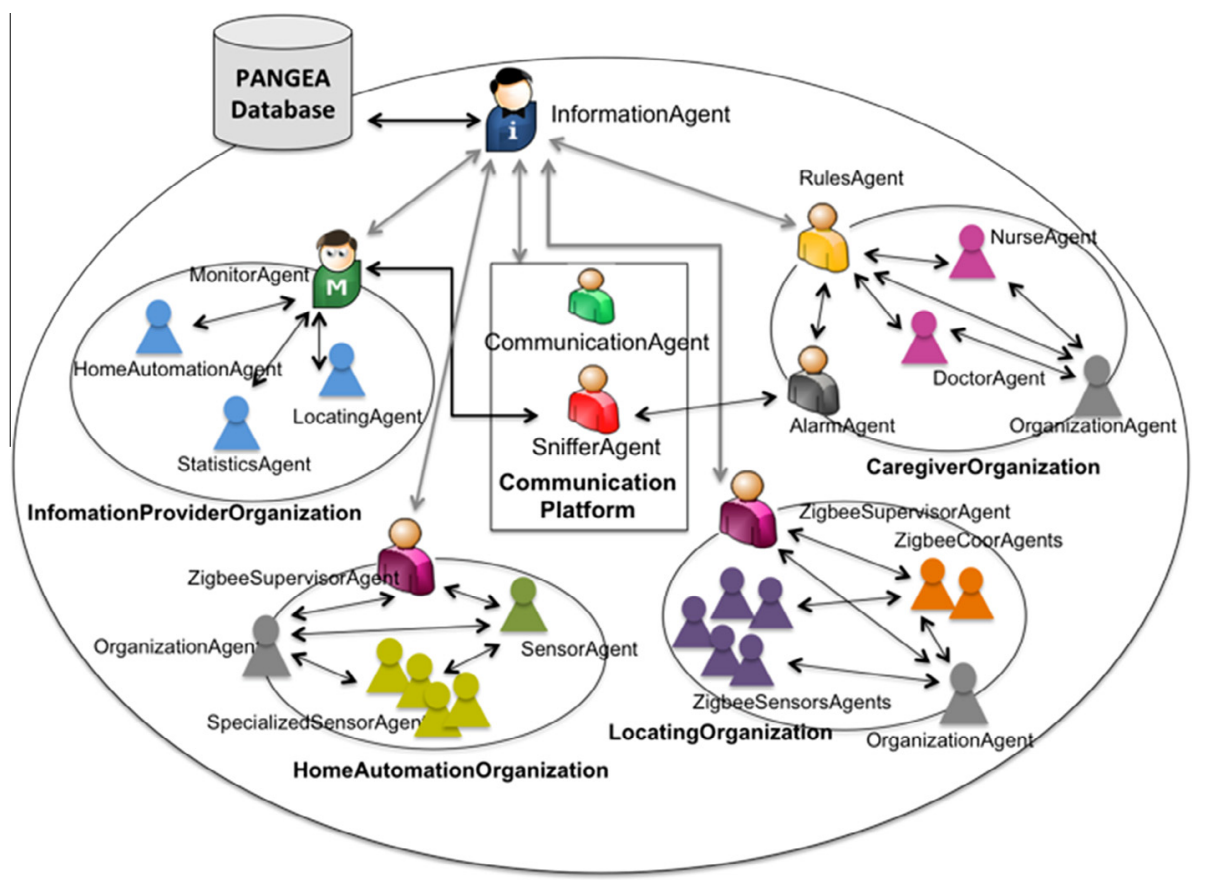

Fig. 11. Overview of the deployed agents in PANGEA.

Residencial La Vega facility has residence areas for the elderly as well as apartments similar to their patients' home. We would like to highlight the level of participation of the facility's medical personnel in implementing the prototype.

In this results section we have included some of the most relevant results obtained in the implementation of this system in the residence. Specifically, in this section, several aspects are important:

- On one hand, the possible functions of the system, showing some of the tools created (web and mobile tools) used by users of the system and results show the fusion of sensor data and information stored in the system in a distributed manner. All these functionalities are implemented with PANGEA, so we must make it clear that these features are just a sample of what could turn out to be because this platform is very easy to scale and the system can be enhance.

- On the other hand, the results of the rule system and the location algorithm used with the sensors installed in the residence are shown. In this sense, favorable results are shown in a possible evolution of the system of rules.

First, we propose to study the reasons for residence and location where they occurred (areas) taking as input data the observed data from the sensors in different areas. In short, put in any space, whether conceptual or geometric, the reasons and the situation inside the residence, integrating them in context, and interpret that situation. The residence is divided into zones (red, blue, yellow or green). Each contains a type of residents. For example, the blue area is indicated for patients with some form of dementia such as Alzheimer's and green area for seniors who can fend for themselves.

About installation, the system services were put in place for some elders, taking into account the considerations of the staff of the residence. The growing use of telemonitoring to support independent living in a residence inevitably carries out many ethical questions regarding privacy. A resident's right to confidentiality must be respected in any feature of healthcare, telemonitoring included. At the resident side, the intrusiveness of the continuing analysis of the resident in his/her own private life should be minimized as much as possible. In this sense, approval for the study was obtained from the Residencial La Vega Committee and the University of Salamanca. Moreover, all personal data were transformed previously to the storage experiment changing them for general identifiers and data encryption and secure methods were applied to ensure confidentiality of the data during transfer over the system. At the monitoring end, access to data was restricted using a hierarchical password system for the persons implied in the experiments.

As a result of the information obtained from both the telemonitoring sensors and those of the caregivers and doctors, the system agents were able to provide a system capable of interacting to control the state of the patient through a web tool.

The information obtained will permit the system to adapt dynamically to the needs of the environment and of the patient. For example, the second graph in Fig. 12 shows how a very different number of assistive actions are taken in the different areas of the residence (red, blue, yellow or green). ${ }^{1}$ The $x$-axis represents the hours in a day and the $y$-axis the number of attendances by falls. The green area underscores the number of falls between the hours of 9 and 12 noon. If we take into account the fact that the green area is where the residents with a specific degree of dependency are located, we can adapt the number of assigned personnel to meet these needs. Additionally, the responsible personnel can use mobile devices to locate patients in real time, plan daily work tasks and respond to alarms. Personnel in the residence can access details regarding an alert and once it has been resolved, they can close the alert with a description of what occurred and the reason for it. Likewise, it is possible to consult the different alerts made in each area with respect to the time or the month they occurred, which is useful information for creating patterns. The graphical representation of this information can be done from the web page or from a mobile device. The operator can also modify the access data and view a summary of their activity. These three functions can be seen in Fig. 13.

The PANGEA multi-agent platform is used to implement the system and the information fusion process. There are several

\footnotetext{
${ }^{1}$ For interpretation of color in Fig. 12, the reader is referred to the web version of this article.
} 

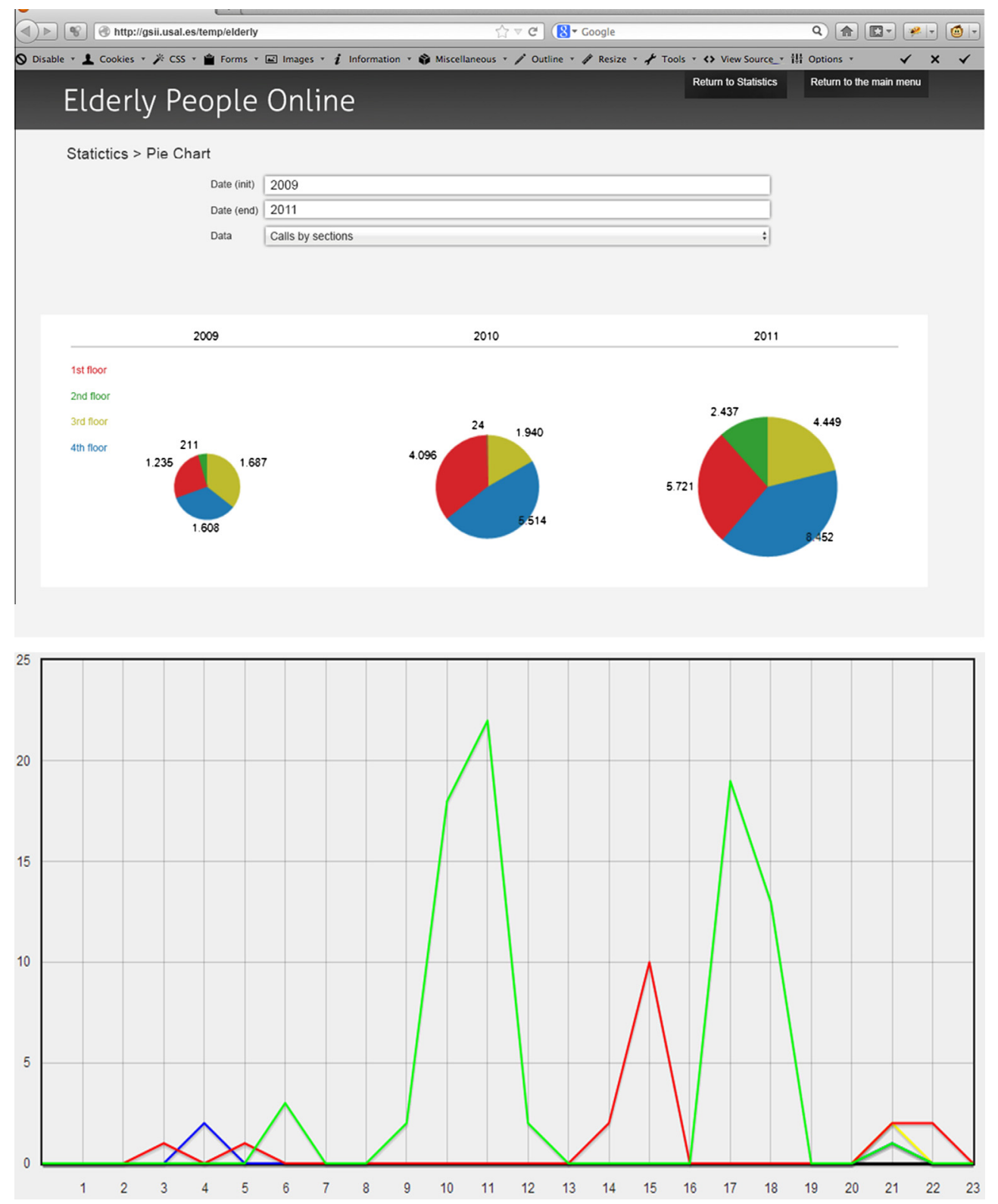

Fig. 12. Web interfaces to query statistics. Top image: Number of calls in the residence by sections. Bottom image: Graph of falls ( $y$ axis) per hour ( $x$ axis).

reasons to prefer such a platform: a multi-agent system represents an advantageous paradigm for the analysis, design and implementation of complex systems. An Information Fusion application is naturally distributed: data sources are distributed; the users use the results produced by the system are distributed, and data processing is performed in a distributed manner. PANGEA provides the infrastructure to create our multi-agent system and also to gather the data, communicate with users, and process data. If data from different sources are private or classified, PANGEA makes it possible to create different forms of access which are not available for centralized processing. Additionally, the system can be large scale in the future, and naturally deconstructed as well; this allows it to takes the most advantage of the benefits provided by the MAS (and PANGEA) approach.

To test the system's performance, we analyzed the precision in locating and detecting anomalous behaviors. The precision of the location algorithm was analyzed by comparing the mean absolute error obtained from applying the proposed procedure to the means obtained from multilateration and signpost. Besides, the Bayesian network was replaced by others classifiers in order to compare the performance. The calculation in the maps of intensities was performed by measuring the steps taken when walking through the different hallways and rooms. In each monitored area, a reference point was used to represent the area, manage the information, and therefore reduce the information that needed to be stored. In each room, a mesh was used to simulate movement patterns and obtain the surface of the room that was measured. The mean absolute error obtained is shown in Table 3.

In order to carry out the alarm detection procedure, the users' movement patterns were stored over a period of one week. During this time, the patterns were classified to include unusual behavior. The number of measurements taken is 983 , following the information shown in Tables 1 and 2. A total of 173 unusual situations were identified. This information was used to create a decision tree that was subsequently used to perform the classification. The rules that were generated are similar in form to the following rule: if User $=13$ and Node $!=13,001$ and sensor $=13,002$ and state $=1$ and measurements $>10$ then alarm $=1$. 

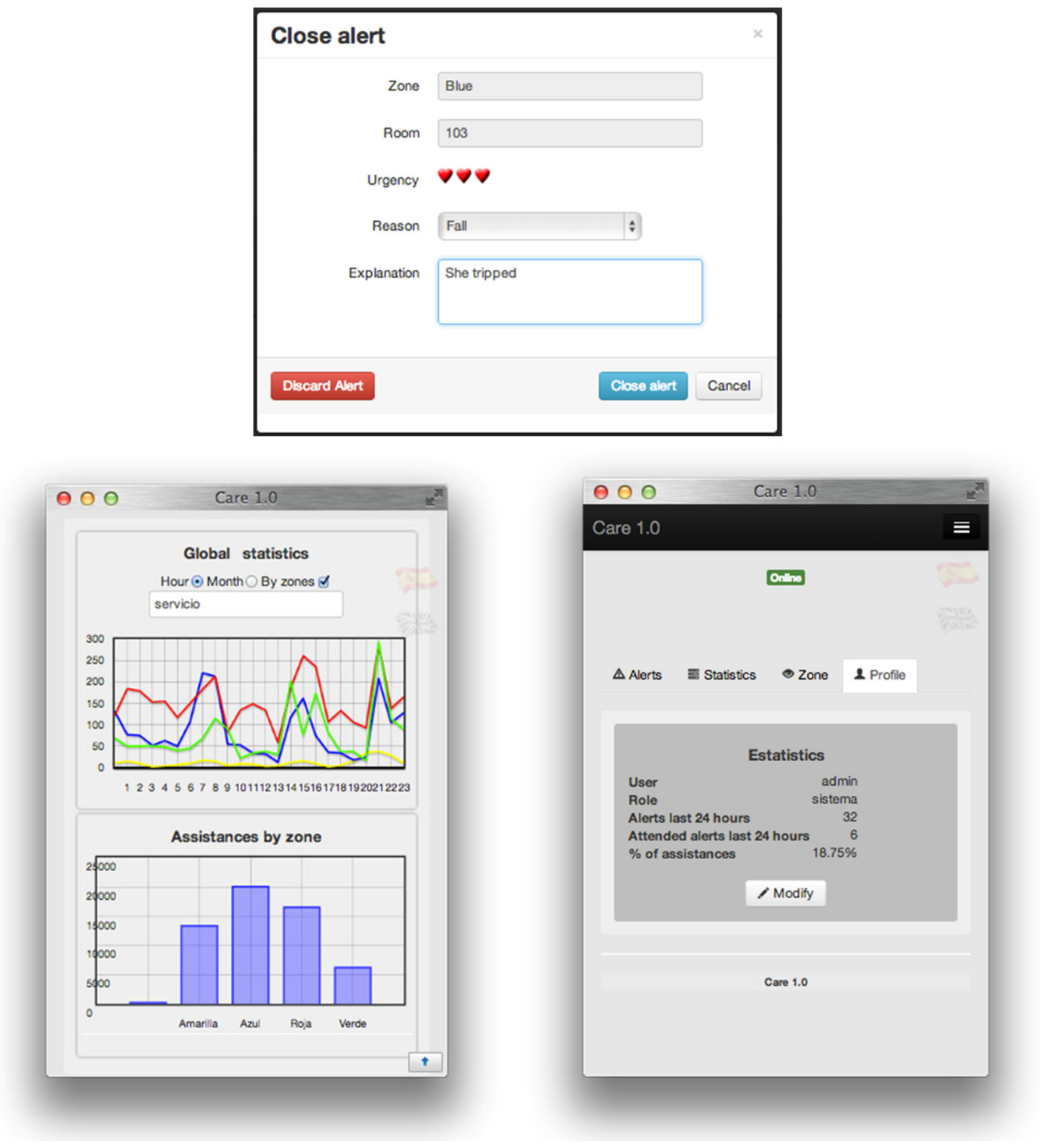

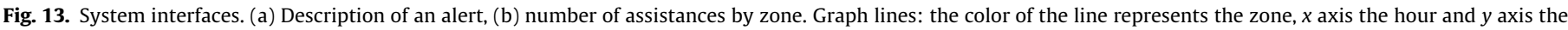

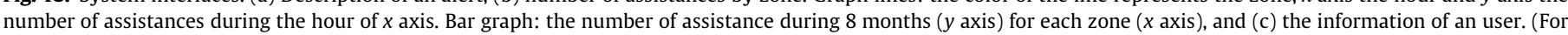
interpretation of the references to color in this figure legend, the reader is referred to the web version of this article.)

Table 3

Error comparison between methods applying maps of intensity and algorithms without applying maps of intensities.

\begin{tabular}{ll}
\hline Model & Mean absolute error \\
\hline Proposed model with Bayesian network & 1.188 \\
Proposed model with J48 & 1.648 \\
Proposed model with SVM & 1.789 \\
Proposed model with IBK & 1.475 \\
Multilateration & 1.981 \\
Signpost & 2.371 \\
\hline
\end{tabular}

The rule refers to user 13 , which is why the sensor identifiers and the nodes in the user's bedroom begin with 13 . When 10 consecutive measurements are detected in a position different from the closest node, which is located in the refrigerator, an alarm will sound. When the rules are detected, as indicated in rule 1 , where all the sensors and the user belong to the same room, they are generalized for all users so that the user ID, sensor and node information are all modified for the other users.
The system was cross validated applying the one-leave-out technique, which resulted in an average prediction rate of $93.5 \%$. The percentage of false negatives rose to $1.2 \%$ and the remainder were false positives.

In order to analyze the evolution of the system, we began with 500 cases and introduced new cases into the system until reaching 983 , as previously indicated. Fig. 14 shows the change in the number of groups in the case base, the evolution of the number of cases and the average number of rules for each group. The diagonal graphs represent the function of density for the indicated variable. For the remaining cells, file $i$, column $j$, the $x$-axis represents the variable indicated in cell $i i$, while in the $y$-axis the variable is associated with cell $j \mathrm{j}$. The dot points represent each element; the red ${ }^{2}$ line represents the tendency according to the represented element, the green line the regression rect. For example, in file 1, column 2 we can see the variation of the average number of rules as the

\footnotetext{
${ }^{2}$ For interpretation of color in Fig. 14, the reader is referred to the web version of this article.
} 


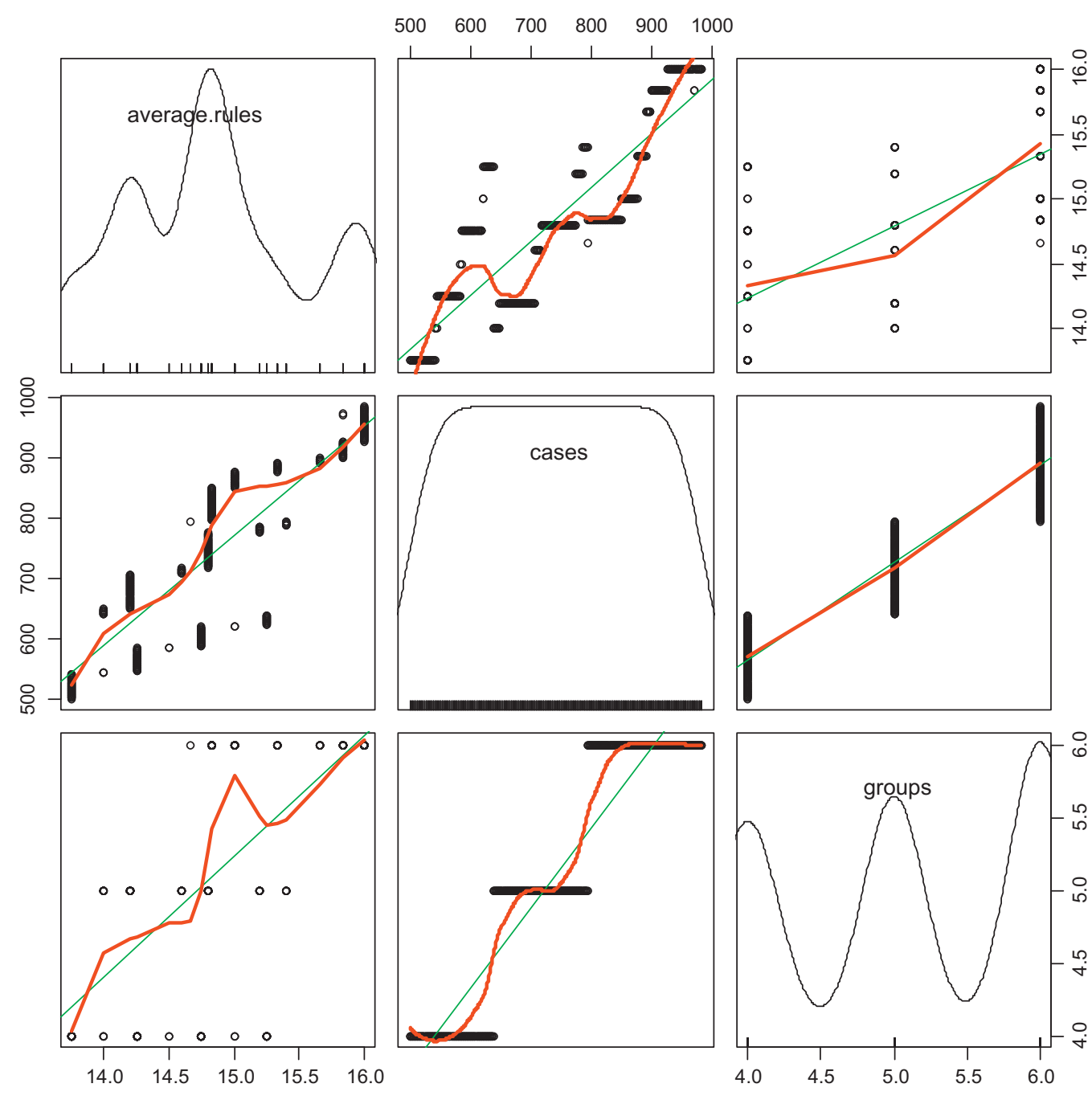

Fig. 14. Evolution of the system.

number of cases increases from 500 to 1000 . We can see in the red line that the tendency increases as the number of cases also increases. However, we can see in the file 1 column 3 that the average number of rules for each group varies between 13 and 16 . Similarly, the number of rules also increases, although to a lesser extent, with the number of groups, as shown in the graph shows.

As we can see in the results, the system proposed in this article exceeds the performance of the system currently used in the La Vega Residence, and improves the existing teleassistance system, providing learning and adaptation capabilities. These systems are currently very limited, as they require the elderly patients to make a conscientious and deliberate effort in their home (for example, pressing the panic button) and do not offer an automated and intelligent detection of high risk situations.

The system proposed in this article implements a significant number of the concepts used in Ambient Intelligence, in an attempt to reduce the interaction of the elderly patient as much as possible and create a much more direct communication with the professional and support staff responsible for providing assistance. The medical personnel at the residence noted the system's ease of use, as well as the support in providing patient care. The aim was to facilitate the daily activities of the elderly patients in a way that is both ubiquitous and intuitive, in addition to optimizing the assistance services and improving quality of life. The proposed system represents an advancement with regard to existing platforms, as shown in its application in the senior care facility case study. It was for this reason that the case study incorporated a set of devices that comprise sensors, id or locating elements, push button actuators and interactive elements such as screens. The study also incorporated a control center that interacts continuously with the system and a data repository, which is very useful for tracing services and the personalization of spaces and services.

\section{Acknowledgements}

This work has been carried out by the project Sociedades Humano-Agente: Inmersion, Adaptacion y Simulacion. TIN201236586-C03-03. Ministerio de Economía y Competitividad. Fondos Feder. Special thanks to Limcasa and Flag Solutions for their support, as well as the technology provided.

\section{References}

[1] R.S. Alonso, D.I. Tapia, G. Villarrubia, J.F. De Paz, Agent technology and wireless sensor networks for monitoring patients in residences and their homes, in: 11th International Conference in Practical Applications of Agents And Multiagent Systems - Workshop on Agents for AAL and E-health, Salamanca, Spain, 2013, pp. 417-418.

[2] E. Aarts, R. Wichert, Ambient Intelligence Technology Guide, Springer Verlag, Berlin, 2009. 
[3] F. Castanedo, M.A. Patricio, J.M. García, J. Molina, Information fusion to improve trajectory tracking in cooperative surveillance multi-agent architecture, Inf. Fusion 11 (2010) 243-255.

[4] Y. Liu, S. Wang, X. Du, A multi-agent information fusion model for ship collision avoidance, in: IEEE International Conference on Machine Learning and Cybernetics, Kunming, China, 2008, pp. 6-11.

[5] A. Pfeffer, S. Das, D. Lawless, B. Ng, Factored reasoning for monitoring dynamic team and goal formation, Inf. Fusion 10 (1) (2009) 99-106.

[6] M. Wooldridge, N.R. Jennings, Agent Theories, Architectures, and languages: a survey, in: ECAI-94 Proceedings of the Workshop on Agent Theories, Architectures, and Languages on Intelligent Agents, Amsterdam, The Netherlands, 1995 , pp. 1-22.

[7] J. Sarangapani, Wireless Ad hoc and Sensor Networks: Protocols, Performance, and Control, first ed., CRC Press, Boca Raton, 2007.

[8] J.F. De Paz, D.I. Tapia, R.S. Alonso, C.I. Pinzón, J. Bajo, J.M. Corchado, Mitigation of the ground reflection effect in real-time locating systems based on wireless sensor networks by using artificial neural networks, J. Knowledge Inf. Syst. 34 (1) (2013) 193-217.

[9] C. Nerguizian, C. Despins, S. Affès, Indoor geolocation with received signal strength fingerprinting technique and neural networks, in: 11th International Conference on Telecommunications, Fortaleza, Brazil, 2004, pp. 866-875.

[10] S. Rodríguez, F. De la Prieta, E. García, C. Zato, J. Bajo, J.M. Corchado, Virtual organizations in information fusion, in: 9th International Conference in Practical Applications of Agents and Multiagent Systems - Special Session on Adaptive Multiagent Systems, Salamanca, Spain, 2011, pp. 195-202.

[11] M. Marin-Perianu, N. Meratnia, P. Havinga, L. de Souza, J. Muller, P. Spiess, S. Haller, T. Riedel, C. Decker, G. Stromberg, Decentralized enterprise systems: a multiplatform wireless sensor network approach, IEEE Wirel. Commun. 14 (2007) 57-66.

[12] M.L. Borrajo, J.M. Corchado, E.S. Corchado, M.A. Pellicer, J. Bajo, Multi-agent neural business control system, Inf. Sci. 180 (6) (2010) 911-927.

[13] C. Zato, G. Villarrubia, A. Sánchez, I. Barri, E. Rubión, A. Fernández, C. Rebate, J.A. Cabo, T. Álamos, J. Sanz, J. Seco, J. Bajo, J.M. Corchado, PANGEA - Platform for Automatic coNstruction of orGanizations of intElligent Agents, in: 9th International Conference on Distributed Computing and Artificial Intelligence, Salamanca, Spain, 2012, pp. 229-239.

[14] CommonWell Project <http://commonwell.eu/index.php> 2010

[15] Monami project <http://www.monami.info/> (accessed December 2013).

[16] DISCATEL <http://www.aeerc.com/teletrabajo.cfm> (accessed December 2013).

[17] INREDIS <http://www.inredis.es/> (accessed December 2013).

[18] INCLUTEC <http://evia.imasdtic.es/es/Inicio/corporativo/grupos_de_trabajo/ inclutec/contenido.aspx> (accessed December 2013).

[19] H. Yu-Jin, K. Ig-Jae, C.A. Sang, K. Hyoung-Gon, Activity recognition using wearable sensors for elder care, in: 2nd International Conference on Future Generation Communication and Networking, Hainan Island, China, 2008, pp. 302-305.

[20] P. George, X. George, P. George, Monitoring and modeling simple everyday activities of the elderly at home, in: 7th IEEE Consumer Communications and Networking Conference, Las Vegas, USA, 2010, pp. 1-5.

[21] W.L. Seon, K. Yong-Joong. L. Gi-Sup, O.C. Byung, L. Nam-Ha, A remote behavioral monitoring system for elders living alone, in: 7th International Conference on Control, Automation and Systems, Seoul, Korea, 2007, pp. 2725-2730.

[22] D. Isern, A. Moreno, Computer-based execution of clinical guidelines: a review, Int. J. Med. Informatics 77 (12) (2008) 787-808.

[23] L. GokceB, A.D. Asuman, A.O. Mehmet, A.T. Ibrahim, A.Y. Mustafa, A.O. Alper, SAPHIRE: a multi-agent system for remote healthcare monitoring through computerized clinical guidelines, in: R. Annicchiarico, U. Cortes, C. Udiales (Eds.), Agent Technology and e-Health, Whitestein Series in Software Agent Technologies and Autonomic Computing, Springer Verlag, Berlin, 2008, pp. 2544.

[24] K.P. Hung, G. Tao, X. Wenwei, P.P. Palmes, Z. Jian, N. Wen Long, W.T. Chee, H.C. Nguyen, Context-aware middleware for pervasive elderly homecare, IEEE J. Sel. Areas Commun. 27 (4) (2009) 510-524.

[25] D. Lymberopoulos, A. Bamis, T. Eixeira, A. Savvides, BehaviorScope: real-time remote human monitoring using sensor networks, in: Proceedings of the International Conference on Information Processing in Sensor, Networks, 2008, pp. 533-534

[26] P. Rashidi, D.J. Cook, Keeping the resident in the loop: Adapting the smart home to the user, IEEE Trans. Syst. Man Cyber. 39 (5) (2009) 949-959.

[27] V.R. Jakkula, D.J. Cook, Using temporal relations in smart environment data for activity prediction, in: Proceedings of the 24th International Conference on Machine Learning, Corvallis, Oregon, 2007, pp. 1-4.

[28] B. Gottfried, H.W. Guesgen, S. Hubner, Spatiotemporal reasoning for smart homes, in Designing Smart Homes, The Role of Artificial Intelligence, Springer, 2008, pp. 16-34.

[29] P. George, X. George, P. George, Monitoring and modeling simple everyday activities of the elderly at home, in: Proceedings of the 7th IEEE Consumer Communications and Networking Conference, 2010, pp. 1-5.

[30] H. Medjahed, D. Istrate, J. Boudy, B. Dorizzi, Human activities of daily living recognition using fuzzy logic for elderly home monitoring, in: Proceedings of the IEEE International Conference on Fuzzy Systems, 2009, pp. 2001-2006.

[31] H. Huo, Y. Xu, H. Yan, S. Mubeen, H. Zhang, An elderly health care system using wireless sensor networks at home, in: Sensor Technologies and Applications SENSORCOMM'09, 3rd International Conference, 2009, pp. 18-23.
[32] H. Alemdar, Cem Ersoy, Wireless sensor networks for healthcare: a survey, Comput. Networks 54 (15) (2010) 2688-2710.

[33] E.L. Waltz, J. Llinas, Multisensor Information Fusion, Artech House, Norwood, USA, 1990.

[34] H. Luo, S. Yang, X. Hu, X. Hu, Agent oriented intelligent fault diagnosis system using evidence theory, Expert Syst. Appl. 39 (3) (2012) 2524-2531.

[35] D.I. Tapia, J.A. Fraile, A. De Luis, J. Bajo, Healthcare information fusion using context-aware agents, in: Manuel Graña Romay, Emilio Corchado, and M. Teresa Garcia Sebastian (Eds.), Proceedings of the 5th International Conference on Hybrid Artificial Intelligence Systems - Volume Part I (HAIS'10), SpringerVerlag, Berlin, Heidelberg, 2010, pp. 96-103.

[36] F. Castanedo, J. García, A.M. Patricio, J.M. Molina, Information fusion to improve trajectory tracking in a cooperative surveillance multi-agent architecture, Inf. Fusion 11 (3) (2009) 243-255.

[37] Y.H. Liu, S.Z. Wang, X.M. Du, A multi-agent information fusion model for ship collision avoidance, in: Proceedings of the International Conference on Machine Learning and Cybernetics, 2008, pp. 6-11.

[38] K. Sycara, R. Glinton, B. Yu, J. Giampapa, S. Owens, M. Lewis, LTC C. Grindle, An integrated approach to high-level information fusion, Inf. Fusion 10 (1) (2009) $25-50$.

[39] S. Rodríguez, Y. De Paz, J. Bajo, J.M. Corchado, Social-based planning model for multiagent systems, Expert Syst. Appl. 38 (10) (2011) 13005-13023.

[40] N. Jennings, M. Wooldridge, Agent Technology: Foundations, Applications and Markets, Springer, Germany, 1998.

[41] J. Ferber, O. Gutknecht, F. Michel, From agents to organizations: an organizational view of multi-agent systems, in: Proceedings of AgentOriented Software Engineering VI, 2003, pp. 214-230.

[42] O. Boissier, B. Gateau, Normative multi-agent organizations: Modeling, support and control, in: G. Boella, L.W.N. van der Torre, H. Verhagen (Eds.) Normative Multiagent Systems, Dagstuhl Seminar Proceedings 07122, Vol. II, Germany, 2007. ISSN: 1862-4405.

[43] V. Dignum, A model for organizational interaction: based on agents, founded in logic, PhD. Thesis, 2004.

[44] J. Pavon, J.J. Gomez-Sanz, Agent oriented software engineering with ingenias, in: V. Marik, J.P. Müller, M. Pechoucek (Eds.), Proceedings of CEECMAS, Heidelberg, Germany, 2003, pp. 394-403.

[45] J.F. Hubner, J.S. Sichman, O. Boissier, Using the Moise+ for a cooperative framework of mass reorganization, in: Proceedings of the 17th Brazilian Symposium on Artificial Intelligence - SBIA'04, 2004, pp. 506-515.

[46] F. Zambonelli, Abstractions and infrastructures for the design and development of mobile agent organizations, in: Proceedings of the AgentOriented Software Engineering II, 2002, pp. 245-262.

[47] M. Esteva, Electronic Institutions: from specification to development Technical University of Catalonia, Spain, 2003 (Ph.D. Thesis).

[48] H.V.D. Parunak, J. Odell, Representing Social Structures in UML, in Wooldridge, M.J., Weiß, G., Ciancarini P. (Eds.), Agent-Oriented Software Eng. II. Lecture Notes in Computer Science, vol. 2222, 2002, pp. 1-16. ISBN: 978-3540-43282-1.

[49] D.L. Hall, J. Llinas, An Introduction to multisensor information fusion, Proc IEEE 85 (1) (1997) 6-23.

[50] J. Bajo, J. Vicente, J.M. Corchado, C. Carrascosa, Y.D. Paz, V. Botti, J.F.D. Paz, An execution time planner for the ARTIS agent architecture, Eng. Appl. Artif. Intell. 21 (2008) 769-784.

[51] S. Pang, T. Ban, Y. Kadobayashi, N. Kasabov, Personalized mode transductive spanning SVM classification tree, Inf. Sci. 181 (2011) 2071-2085.

[52] R.S. Alonso, D.I. Tapia, J. Bajo, Ó. García, J.F. de Paz, J.M. Corchado, Implementing a hardware-embedded reactive agents platform based on a service-oriented architecture over heterogeneous wireless sensor networks, Ad Hoc Netw. 11 (1) (2013) 151-166.

[53] J.F. de Paz, D.I. Tapia, R.S. Alonso, C.I. Pinzón, J. Bajo, J.M. Corchado, Mitigation of the ground reflection effect in real-time locating systems based on wireless sensor networks by using artificial neural networks, Int. J. Knowledge Inf. Syst. 34 (1) (2013) 193-217.

[54] D.I. Tapia, J.A. Fraile, S. Rodríguez, R.S. Alonso, J.M. Corchado, Integrating hardware agents into an enhanced multi-agent architecture for Ambient Intelligence systems, Inf. Sci. 222 (10) (2013) 47-65.

[55] C. Zato, A. Sanchez, G. Villarrubia, S. Rodriguez, J.M. Corchado, J. Bajo, Platform for building large-scale agent-based systems, in: IEEE Conference on Evolving and Adaptive Intelligent Systems, Madrid, Spain, 2012, pp. 69-73.

[56] D.I. Tapia, O. García, R.S. Alonso, F. Guevara, J. Catalina, R.A. Bravo, J.M. Corchado, The n-Core Polaris Real-Time Locating System at the EvAAL Competition, in: Evaluating AAL Systems Through Competitive Benchmarking, Indoor Localization and Tracking Communications in Computer and Information Science, 2012, pp. 92-106.

[57] D.I. Tapia, F. De la Prieta, S. Rodríguez, J. Bajo, J. M. Corchado, Organizations of Agents in Information Fusion Environments, in: L. Antunes, H. S. Pinto (Eds.), Progress in Artificial Intelligence - 15th Portuguese Conference on Artificial Intelligence - EPIA 2011, Lisbon, Portugal, 2011, pp. 59-70.

[58] J.M. Corchado, J. Bajo, D.I. Tapia, A. Abraham, Using heterogeneous wireless sensor networks in a telemonitoring system for healthcare, in: IEEE Transactions on Information Technology in Biomedicine - Special Issue: Affective and Pervasive Computing for Healthcare, 2009, pp. 663-670.

[59] J.F. De Paz, D.I. Tapia, R.S. Alonso, C.I. Pinzón, J. Bajo, J.M. Corchado, Mitigation of the ground reflection effect in real-time locating systems based on wireless sensor networks by using artificial neural networks, Knowl. Inf. Syst. 34 (2013) 193-217. 
[60] R.R. Bouckaert, Bayesian Belief Networks: from Construction to Inference, Utrecht, Netherlands, 1995.

[61] T. Verma, J. Pearl, An algorithm for deciding if a set of observed independencies has a causal explanation, in: Proceedings of the Eighth Conference on Uncertainty in Artificial Intelligence, 1992, pp. 323-330.

[62] N. Friedman, D. Geiger, M. Goldszmidt, Bayesian Network Classifiers, Machine Learning 29 (1997) 131-163.
[63] ZigBee Standards Organization, ZigBee Specification Document 053474r13, 2006.

[64] Drools. The Business Logic Integration Platform <http://www.jboss.org/drools/ $>$ (accessed December 2013)

[65] B. Berstel, Extending the RETE algorithm for event management, in: Temporal Representation and Reasoning - Proceedings - Ninth International Symposium, 2002, pp. 49-51. 